\title{
Modelización de línea aérea de distribución rural para evaluación del desempeño ante descargas atmosféricas aplicado al software EMTP RV
}

\section{Rural distribution overhead line modeling for performance assess- ment for atmospheric discharge applied to EMTP RV software}

\author{
Abél C. Catay B. ${ }^{(1)}$ \\ ${ }^{(1)}$ Facultad de Ingeniería Eléctrica y Electrónica de la Universidad Nacional del Centro del Perú \\ Email: acatay@uncp.edu.pe
}

\begin{abstract}
RESUMEN
El presente trabajo de investigación tuvo como problema general el cómo modelar la línea aérea de distribución rural para evaluar su desempeño ante descargas atmosféricas aplicado al software EMTP RV. Se utilizó el método de modelización y simulación, considerando el procedimiento secuencial: Descripción del problema, construcción de un modelo conceptual, construcción del modelo para computadora, simulación de diferentes escenarios actuando sobre las variables independientes, análisis de sensibilidad y presentación de resultados. El método descrito se aplicó al software EMTP RV. En el procedimiento metodológico, se emplearon, como materiales, las normas vigentes de electrificación rural en el Perú de la Dirección General de Electricidad del MEM. El tipo de investigación es aplicada y el nivel es correlacional. Se concluye que, el modelo línea aérea de distribución rural aplicado al software EMTP RV implementado, permite evaluar íntegramente su desempeño ante descargas atmosféricas sólo en impactos directos. El modelamiento del soporte, como impedancia de impulso y el aislamiento de la línea aérea de distribución rural con sus valores de CFO (Critical Impulse Flashover Voltage), logra respuestas correctas en la evaluación del desempeño ante descargas atmosféricas directas. El modelo de la línea aérea de distribución rural implementado, permite evaluar alternativas de mejora como la inclusión de cable de guarda y valores diferentes de resistencia de puesta a tierra.
\end{abstract}

Palabras clave: Líneas de distribución - descarga atmosférica - EMTP RV - modelización.

\begin{abstract}
The present research work had as general problem regarding how to model the rural distribution airline to evaluate its performance against atmospheric discharges applied to the EMTP RV software. The modeling and simulation method was used, considering the sequential procedure: Description of the problem, construction of a conceptual model, construction of the computer model, simulation of different scenarios acting on the independent variables, sensitivity analysis and presentation of results. The method described was applied to the EMTP RV software. In the methodological procedure, the current rules of rural electrification in Peru of the General Directorate of Electricity of the MEM were used as materials. The type of research is applied and the level is correlational. It is concluded that the rural distribution model applied to the implemented EMTP RV Software allows to fully evaluate its performance against atmospheric discharge only for direct impacts. The modeling of the pole as impulse impedance and the insulation of the rural distribution airline with its CFO (Critical Impulse Flashover Voltage) values obtain correct answers in the evaluation of the performance to direct atmospheric discharges. The model of the implemented rural distribution airline, allows to evaluate alternatives of improvement like the inclusion of guard wire and different values of earth resistance.
\end{abstract}

Key words: Distribution lines - atmospheric discharge EMTP RV - modeling. 


\section{INTRODUCCIÓN}

El problema general planteado para el desarrollo del presente trabajo de investigación es: ¿Cómo modelar la línea aérea de distribución rural para evaluar su desempeño ante descargas atmosféricas aplicado al software EMTP RV? Además, se han considerado dos problemas específicos, los cuales son: ¿Cómo modelar el soporte y el aislamiento de la línea aérea de distribución rural a fin de lograr respuestas correctas en la evaluación del desempeño ante descargas atmosféricas? ¿Cómo evaluar, en el modelo de la línea aérea de distribución rural aplicada al software EMTP RV, alternativas de mejora en el desempeño ante descargas atmosféricas?

Actualmente, en nuestro país la expansión y los proyectos de electrificación rural se ejecutan a niveles de tensión normalizadas de 22,9 kV y 22,9/13,2 kV con soportes de madera y concreto. Las líneas se caracterizan por ser trifásicos, bifásicos, monofásicos con retorno por el neutro y retorno total por tierra. Estas líneas, durante su operación, se exponen a sobretensiones de origen atmosférico porque las zonas de sierra y selva del Perú tienen alto nivel ceráunico.

Diferentes diagnósticos afirman que los menores niveles de calidad de suministro se encuentran en las zonas rurales y las sobretensiones a causa de las descargas atmosféricas directas e indirectas son las que ocasionan los mayores números y tiempos de salidas de servicio de las redes eléctricas rurales.

Por las razones explicadas, el presente trabajo de investigación busca cumplir con los objetivos de: modelar la línea aérea de distribución rural para evaluar su desempeño ante descargas atmosféricas aplicado al software EMTP RV; modelar el soporte y el aislamiento de la línea aérea de distribución rural a fin de lograr respuestas correctas en la evaluación del desempeño ante descargas atmosféricas; y evaluar, en el modelo de la línea aérea de distribución rural aplicado al software EMTP RV, alternativas de mejora en el desempeño ante descargas atmosféricas.

\section{MATERIALES Y METODOS}

\section{Lugar de ejecución del trabajo}

El presente trabajo de investigación se ha desarrollado en la ciudad de Huancayo y en los ambientes de la Facultad de Ingeniería Eléctrica y Electrónica de la Universidad Nacional del Centro del Perú.

\section{Procedencia del material utilizado}

Como materiales, se utilizaron las normas vigentes de la Dirección General de Electricidad del Ministerio de Energía y Minas [1] [7], de donde se obtienen los parámetros de los postes para el cálculo de la impedancia característica con la propuestas de Sargent \& Darveniza, y el modelo IEEE/CIGRE del IEEE Std. 1410-2010 [ ]; se describe y compara el modelo de la onda de impulso de la corriente de rayo con el implementado en el EMTP RV. Como herramientas para la modelización y simulación de la línea aérea de distribución rural se utiliza el Software para simulación y análisis de transitorios en sistemas de potencia EMTP RV versión 2.0.2. Para cálculos matemáticos y gráficos adicionales Matlab R2012a y Excel 2013 en un computador personal CORE i5, 2.30 GHz y 8 GB de memoria $R A M$ y sistema operativo Windows 7 Ultimate Service Pack 1.

\section{Población}

La población de estudio está constituida por líneas aéreas de distribución.

\section{Muestra}

La muestra considerada en el estudio es líneas aéreas de distribución rural pertenecientes a los sistemas eléctricos rurales.

\section{Metodología de análisis}

El método utilizado para desarrollar el presente proyecto de investigación es el de modelización y simulación. El método tiene el siguiente procedimiento secuencial: Descripción del problema, construcción de un modelo conceptual, construcción del modelo para computadora, simulación de diferentes escenarios actuando sobre las variables independientes, análisis de sensibilidad y presentación de resultados [4]. El método descrito se aplica al software EMTP RV.

\section{Diseño de la investigación Tipo de investigación}

Por el tipo de investigación, el presente estudio, reúne las condiciones metodológicas de una investigación aplicada, en razón que se utilizaron conocimientos de las ciencias empleadas en la ingeniería eléctrica, a fin de aplicarlas en la modelización de línea aérea de distribución rural para evaluación del desempeño ante descargas atmosféricas por lo que se utilizó un software de especialidad.

\section{Nivel de investigación}

La investigación reúne características de diseño correlacional y explicativo. Se estudia las relaciones entre variables dependientes e independientes, se busca el por qué de los hechos, estableciendo relaciones de causa - efecto.

\section{RESULTADOS}

Se describe la modelización de los elementos de la red rural considerando los parámetros teóricos y normativos, se compara y se relaciona con los elementos implementados en el EMTP RV.

\section{Modelización del soporte de madera}

En 1968, Sargent y Darveniza [3] [6], publican un impor- 
tante paper en el cual calculan la impedancia de impulso equivalente de una torre para una variedad de formas y comparado estos con actuales mediciones en nanosegundos en modelos geométricos. Los resultados relevantes de este análisis se presentan en la Figura 1. El valor $2 r$ en el perfil de clase 2, es el diámetro de la cabeza hacia abajo para el caso de un poste de madera o el diámetro del poste de metal si es una estructura de metal. La impedancia característica para el perfil de la clase 3 es una aproximación de la impedancia característica de un cilindro recto. Impedancias características de otras clases de estructuras pueden determinarse bien mediante cálculos de las clases 1, 2 ó 3, o mediante mediciones en modelos geométricos. Los valores suelen variar de 100 a $300 \Omega$.

Figura 1. Aproximaciones para impedancias de impulso de torres. [3] [6]

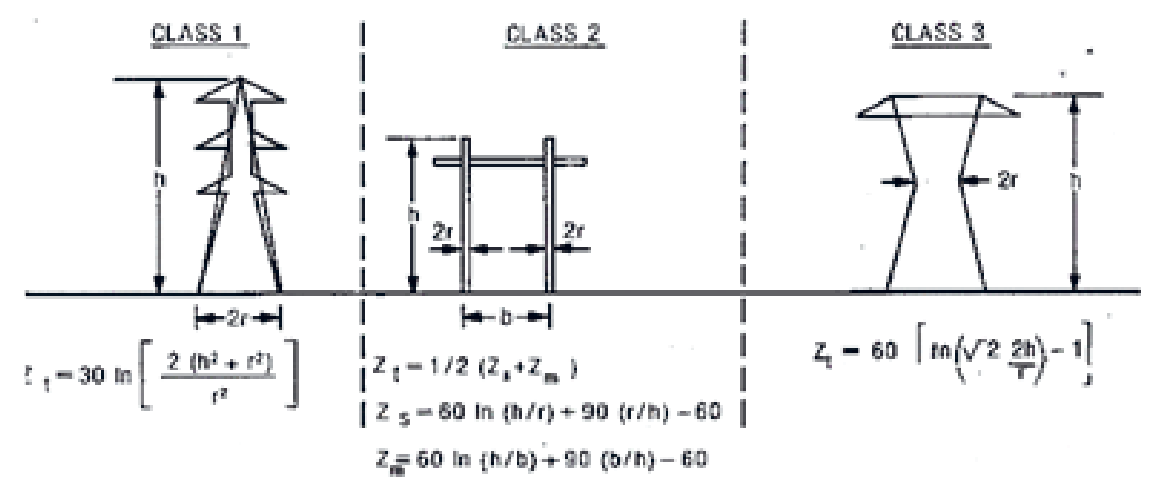

FOA EACH CASE, THE TAAVEL TIME T FAOM TOWEA TOP TO GROUND IS: $\boldsymbol{T}-\mathrm{h} / 300$, $\mu \mathrm{t}$

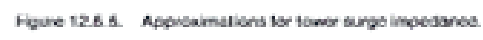

Para un solo poste, en el análisis de la clase 2, la impedancia mutua $\mathrm{Zm}$ es cero. Entonces, la expresión para el cálculo de la impedancia de impulso de un soporte vertical empotrado sobre un suelo plano está dado por:

$$
Z s=60 \ln \left(\frac{h}{r}\right)+90\left(\frac{r}{h}\right)-60[\Omega]
$$

En la norma DGE. "Especificaciones técnicas para el suministro de materiales y equipos de líneas y redes pri- marias para electrificación rural" [7] se especifíca que se debe utilizar poste de madera de longitudes de $11 \mathrm{~m}, 12$ m y 13 m de clases 5 y 6 .

Con las anteriores consideraciones y asumiendo que el poste tiene la forma de un cilindro representado por un radio promedio, de la cabeza y al nivel de la línea de tierra del poste se utiliza la propuesta de Sargent y Darveniza para calcular la impedancia de impulso con la ecuación (4.1) para las tres longitudes y dos clases de postes especificados, los resultados se muestran en la Tabla 01 .

Tabla $\mathbf{N}^{\circ}$ 01. Impedancia de impulso. Modelo Sargent y Darveniza

\begin{tabular}{cccccccccccc} 
& Clase & \multicolumn{2}{c}{$\mathbf{5}$} & & & & & $\mathbf{6}$ & \\
& Grupo & A & B & C & D & E & A & B & C & D & E \\
\hline $\begin{array}{c}\text { Longitud poste } \\
\text { (m) - Largo }\end{array}$ & 9.30 & 221.3 & 219.3 & 216.1 & 212.8 & 208.7 & 228.9 & 225.8 & 223.0 & 219.2 & 214.7 \\
empotramiento & 10.20 & 225.7 & 223.6 & 220.6 & 217.3 & 212.8 & 233.1 & 230.2 & 226.8 & 223.6 & 219.2 \\
$(m)$ & 11.10 & 229.7 & 227.1 & 224.2 & 220.9 & 217.0 & 236.9 & 234.0 & 230.7 & 227.6 & 223.2
\end{tabular}

Otra propuesta para modelar la impedancia de impulso es la fórmula propuesta por la IEEE/CIGRE. [8]

$$
Z t=60 \operatorname{Ln}\left\{\operatorname{Cot}\left[0,5 \operatorname{Tan}^{-1}\left(\frac{R}{h}\right)\right]\right\} \Omega
$$

Donde:

- $R=\left(r_{1} h_{1}+r_{2} h+r_{3} h_{2}\right) / h$ es el radio equivalente de la torre representado por un cono truncado, $h=h_{1}$ $+h_{2}$

- $r_{1}, r_{2}, r_{3}$ es el radio en la parte superior, sección media $\mathrm{y}$ base $[\mathrm{m}]$.
- h1 es la altura desde la sección media hasta la parte superior $[\mathrm{m}]$

- h2 es la altura desde la base hasta la sección media $[m]$

Cuando la torre no es un cono, sino un cilindro; en este caso, la anterior ecuación se reduce a:

$$
Z t=60 \operatorname{Ln}\left(\frac{h}{r}\right) \Omega
$$

Donde $r$ es el radio de un cilindro que representa a la torre. 
Figura 2. Modelo de torre propuesto por IEEE/CIGRE. [8]

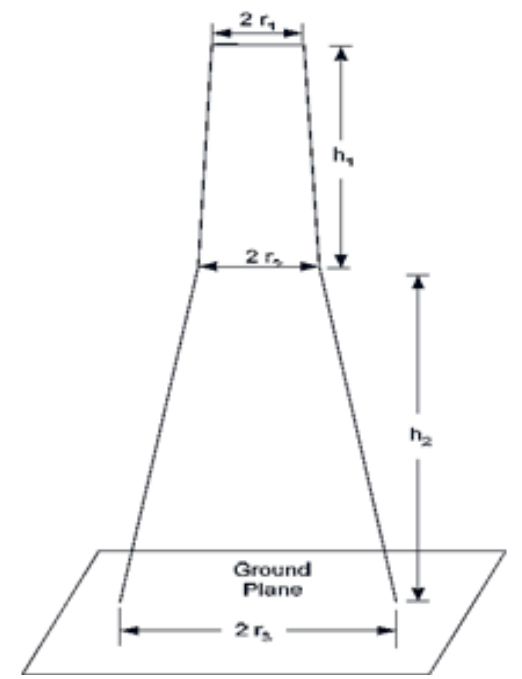

Con el modelo propuesto por IEEE/CIGRE y utilizando la ecuación (4.2) y las tres longitudes y dos clases de postes especificados, se calculan las impedancias de impulso. Los resultados se muestran en la Tabla 2.

El poste de la línea aérea de distribución rural será modelizada como la impedancia característica de una línea de transmisión monofásica de parámetros constantes. La línea de parámetros constante $(C P)$ se clasifica como un modelo de línea de transmisión independiente de la frecuencia. Su principal ventaja es la velocidad de cálculo. Es menos precisa que la línea dependiente de la frecuencia y modelos de cable, pero puede ser utilizado con éxito en el análisis de problemas con reducida dispersión de frecuencia.

Los parámetros de la línea $C P$ se calculan a una frecuencia dada y es por eso que se etiqueta como una línea independiente de la frecuencia.

Tabla $\mathbf{N}^{\circ} 02$ Impedancia de impulso. Modelo IEEE/CIGRE

\begin{tabular}{ccccccccccccc} 
& CLASE & \multicolumn{1}{c}{$\mathbf{5}$} & & & & & $\mathbf{6}$ & \\
& GRUPO & A & B & C & D & E & A & B & C & D & E \\
\hline $\begin{array}{c}\text { Longitud poste } \\
\text { (m) - Largo }\end{array}$ & 9.30 & 280.50 & 278.33 & 275.23 & 271.80 & 267.10 & 288.12 & 285.07 & 282.17 & 278.33 & 273.74 \\
empotramiento & 10.2 & 284.95 & 282.82 & 279.77 & 276.40 & 271.89 & 292.42 & 289.44 & 286.04 & 282.82 & 278.30 \\
$(m)$ & 11.10 & 288.95 & 286.35 & 283.38 & 280.08 & 276.10 & 296.28 & 293.35 & 290.02 & 286.86 & 282.42
\end{tabular}

Por tanto, los parámetros de ingreso para modelizar el poste de la línea aérea de distribución son: El valor de la impedancia característica calculado con la propuesta de Sargent y Darveniza, y el modelo IEEE / CIGRE presentados en las tablas 01 y 02 ; la velocidad de propagación del impulso a través de la $C P$ que se considera la velocidad de la luz $3 \times 108 \mathrm{~m} / \mathrm{s}$; y la longitud efectiva del poste que representara la longitud de la línea $C P$.

\section{Modelización del aislador}

A pesar que el ingeniero de diseño puede estar más familiarizado con el nivel básico de aislamiento al impulso (BIL) de una determinada combinación de materiales aislantes, los análisis y resultados del IEEE Std 1410 - 2010 se dan en términos del CFO de estas combinaciones. El CFO se define como el nivel de tensión en el que estadísticamente hay una probabilidad del 50\% de flameo y una probabilidad del 50\% de soportar el esfuerzo de tensión. Este valor de resistencia al flameo se define en pruebas de laboratorio, y tiende a tener una distribución gaussiana estrecha en comparación con las amplias distribuciones log-normal de estrés basado en parámetros de corriente de rayos. ${ }^{1} \mathrm{Si}$ se supone una distribución gaussiana de la resistencia de flameo, entonces cualquier probabilidad específica de soportar podrá ser calculado estadísticamente a partir del valor $C F O$ y la desviación estándar. [2]

Los valores del CFO de los aisladores son especificados por

1 Tabla 1 - Recomendaciones de parámetros de corriente de rayos (Grupo de Trabajo CIGRÉ 33.01 [B27]). IEEE Std 1410 - 2010. el fabricante en los catálogos diferente a los valores del BIL. Entonces, la modelización del aislador en el EMTP RV se realiza mediante la utilización del bloque Flashover switch. Este dispositivo simula un interruptor de flameo basado en una condición de voltaje que es el CFO del aislador. Si la tensión que aparece a causa del rayo supera el CFO del aislador el Flashover switch se cortocircuita simulando un flameo fase tierra o fase fase.

\section{Modelización de las puestas a tierra}

Los criterios para el dimensionamiento de las puestas a tierra en líneas de media tensión, incluyendo las de electrificación rural son los siguientes: [9]

- Seguridad de las personas

- Operación del sistema

- Descargas atmosféricas

- Facilidad para el recorrido a tierra de las corrientes de fuga La norma DGE. "Bases para el diseño de líneas y redes primarias para electrificación rural”, prescribe que para sistemas con neutro corrido la resistencia de puesta a tierra equivalente de todas las puestas a tierra debe ser menor o igual a $3 \Omega$. En sistemas sin neutro corrido y con retorno total por tierra considera que la resistencia de puesta a tierra que realmente importa es de la sub estación de distribución porque la resistencia de puesta a tierra en la sub estación de potencia es generalmente menor a $3 \Omega$. La norma indica que en líneas primarias, sin cable de guarda, se puede aceptar resistencias de puesta a tierra de hasta 
$500 \Omega$; y en líneas de zonas de alto nivel ceráunico con cables de guarda las resistencias de puesta a tierra deberá estar comprendida entre 10 y $15 \Omega$.

Finalmente, la norma indica que para sub estaciones de distribución trifásicas y monofásicas conectadas entre fases (bifásicas) la resistencia de puesta a tierra debe ser menor a $25 \Omega$ y en transformadores monofásicos de sistemas con retorno total por tierra, la norma especifíca las máximas resistencias de puestas a tierra de acuerdo a la potencia de los transformadores, de acuerdo a la siguiente tabla.

Tabla $\mathbf{N}^{\circ}$ 03. Máximas resistencias de puesta a tierra de acuerdo a la potencia del transformador

$\begin{array}{cc}\begin{array}{c}\text { Potencia de transformador } \\ \text { kVA }\end{array} & \begin{array}{r}\text { Resistencia de puesta a tierra } \\ \text { (0) }\end{array} \\ 5 & 25 \\ 10 & 25 \\ 15 & 20 \\ 25 & 15\end{array}$

Fuente: Norma DGE. "Especificaciones Técnicas para el Suministro de Materiales y Equipos de Líneas y Redes Primarias para Electrificación Rural”.

La modelización de las puestas a tierra en el EMTP RV se realiza mediante la utilización del bloque RLC device. Este dispositivo es configurado solamente como un resistor monofásico y está conectado entre los puntos a potencial cero de la estructura de la línea (conductor de guarda, elementos metálicos, pararrayos) y el bloque Ground que establece un perfecto potencial cero.

\section{Modelización del rayo}

La modelización del rayo se basa en la propuesta del CIGRÉ Working Group 33.01. ${ }^{2}$ La forma de la corriente de impulso se considera que es una fuente de corriente, en la figura 3 y tabla 04 se describen la forma y los parámetros del rayo representados por una corriente de impulso. [2]

Figura 3. Descripción de los parámetros de forma de onda de la corriente de rayo

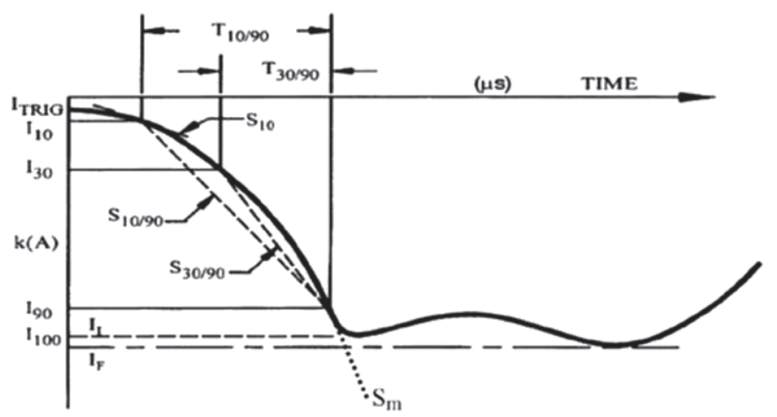

Fuente: Norma DGE. "Especificaciones Técnicas para el Suministro de Materiales y Equipos de Líneas y Redes Primarias para Electrificación Rural”.

En la literatura de la especialidad este modelo se define como la onda normalizada de impulso de 1,2/50 $\mu$ s que se utiliza en los estudios de coordinación de aislamiento y selección de pararrayos.

El EMTP $R V$ ha modelado la propuesta de CIGRE, ello se muestra en figura 4 donde Imax es el valor de la intensidad de corriente del rayo, esta fuente de corriente debe ser implementada en paralelo con un resistor de $1 \mathrm{k} \Omega$ de valor conectándose en el bloque de simulación

Tabla $\mathbf{N}^{\circ}$ 04. Parámetros de la onda de impulso de la corriente de rayo

\begin{tabular}{|c|c|}
\hline $\begin{array}{l}\text { Parameter } \\
\text { in Fig. } 3.3\end{array}$ & Description \\
\hline 110 & $10 \%$ intercept along the stroke current waveshape \\
\hline 130 & $30 \%$ intercept along the stroke current waveshape \\
\hline 190 & $90 \%$ intercept along the stroke current waveshape \\
\hline $1100=1 /$ & Initial peak of current \\
\hline IF & Final (global) peak of current (same as peak current without an adjective) \\
\hline T10/90 & Time between /10 and /90 intercepts on the wavefront \\
\hline T30/90 & Time between $/ 30$ and $/ 90$ intercepts on the wavefront \\
\hline$S_{10}$ & Instantaneous rate-of-rise of current at $/ 10$ \\
\hline$S_{10 / 90}$ & Average steepness (through /10 and /90 intercepts) \\
\hline$S_{30 / 90}$ & Average steepness (through /30 and /90 intercepts) \\
\hline$S_{m}$ & Maximum rate-of-rise of current along wavefront, typically at I90 \\
\hline$t_{d} 10 / 90$ & Equivalent linear wavefront duration derived from IF / S10/90 \\
\hline$t_{d} 30 / 90$ & Equivalent linear wavefront duration derived from IF / S30/90 \\
\hline$t_{m}$ & Equivalent linear waveform duration derived from $I F / S_{m}$ \\
\hline QI & Impulse charge (time integral of current) \\
\hline
\end{tabular}

Fuente: Norma DGE. "Especificaciones Técnicas para el Suministro de Materiales y Equipos de Líneas y Redes Primarias para Electrificación Rural”

2 CIGRÉ Working Group 33.01, Guide to procedures for estimating the lightning performance of transmission lines, CIGRÉ Technical Brochure no. 63 , October 1991. 
final a un elemento de la línea de distribución como cable de guarda, conductor o cabeza de poste.

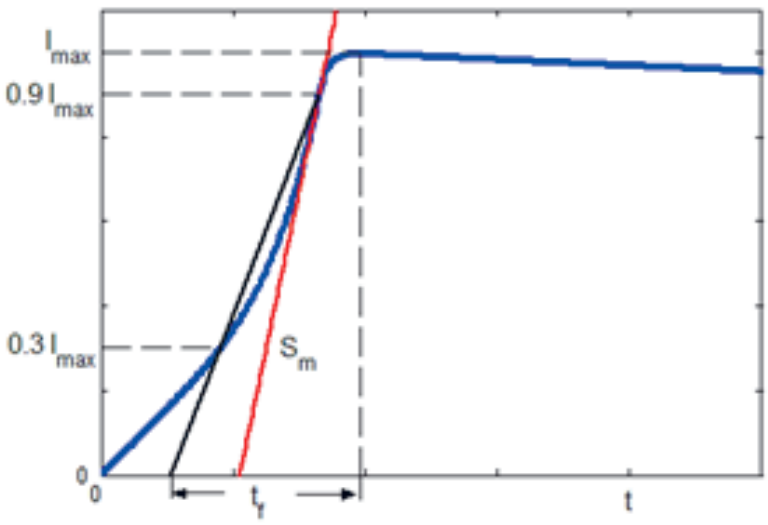

Figura 4. Modelo de corriente de impulso de rayo implementado en EMTP RV

\section{Modelización de la línea}

La línea es modelada utilizando el bloque frequency dependent line: FD m-phase. Este modelo es más preciso que el modelo de línea Constant Parameters (CP). Sin embargo, es computacionalmente más lento. A diferencia del modelo de línea $\mathrm{CP}$, el modelo de línea dependiente de la frecuencia (FD) tiene en cuenta la dependencia de la frecuencia de R' y L' (resistencia en serie e inductancia de la línea por unidad de longitud).

Los datos de la línea se obtienen del dispositivo "Line Data” en la librería lines.clf. Datos de la geometría de la disposición de los conductores, tipo de conductor, numero de fases; con ello el dispositivo Line Data calcula la impedancia de la línea y lo asocia al bloque FD m-phase donde se considera la longitud de la línea.

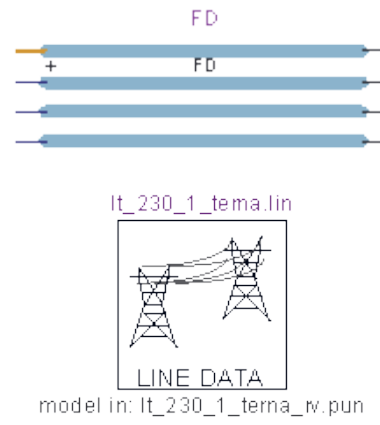

Figura 5. Bloque Frequency dependent line: FD m-phase y dispositivo "Line Data" implementado en EMTP RV

Se enumeran las variables de simulación y los parámetros considerados en la red de distribución:

- Longitud de poste: $13 \mathrm{~m}$.

- Vano promedio: $180 \mathrm{~m}$.

- Resistividad eléctrica del suelo: $200 \mathrm{Ohm}-\mathrm{m}$

- Sección de conductor AAAC: $35 \mathrm{~mm} 2$

- Aislador tipo pin ANSI 56-2; CFO $175 \mathrm{KV}$ positivo

- Resistencia de puesta a tierra $25 \mathrm{Ohm}$

- Delta del tiempo de simulación: $15 \mathrm{~ns}$
- Tiempo de simulación: $100 \mu \mathrm{s}$

- Intensidades de rayo: 0,$5 ; 1 ; 3 ; 5 ; 10 ; 20 ; 30 ; 50 ; 100$ $k A$.

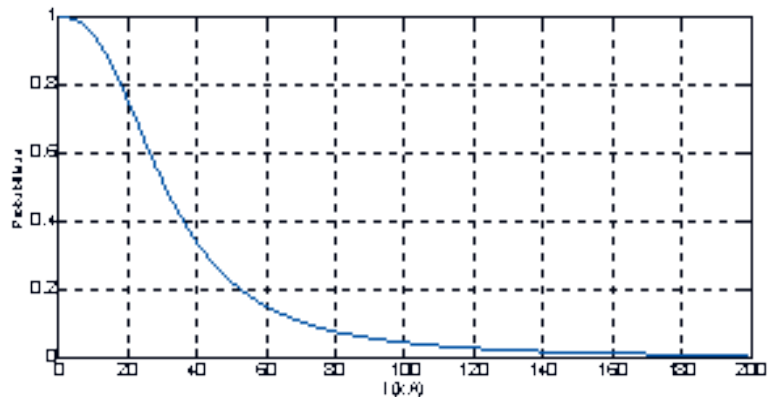

Figura 10. Gráfica de la distribución probabilística de los valores pico de corriente dado por la ecuación (2)

Con las anteriores consideraciones y parámetros se han simulado descargas directas del rayo sobre el conductor más expuesto de la red, siendo la fase $B$ o el conductor de guarda. Los diferentes resultados de descarga disruptiva en los aisladores (flashover) se muestran en los anexos, los cuales se interpretan y discuten en la siguiente sección.

\section{DISCUSIÓN}

De acuerdo a la ecuación (2) y figura 10, la probabilidad que ocurra un rayo con una intensidad igual o mayor a 1 $k A$ es de $99,98 \%$, con los resultados de las simulaciones realizadas para el modelo de red recomendado en la norma $D G E$. "Bases para el diseño de líneas y redes primarias para electrificación rural" [9]; se concluye que, todas las descargas tipo rayo que impacten directamente en algún conductor de fase producirán descarga disruptiva en los aisladores.

La evaluación de la inclusión del conductor neutro del sistema como mejora demuestra que el conductor neutro, ubicado en la parte inferior de las tres fases, no contribuye en reducir el flameo en los aisladores ante descargas directas tipo rayo. En esta valoración se consideró la reducción del valor de la resistencia de puesta a tierra del conductor neutro en cada poste.

Se observa que la inclusión de un conductor de guarda ubicado a un metro por encima de la fase B de la estructura original hace que la red pueda soportar hasta 41 $k A$ de descarga tipo rayo directo; y si además, se incluye el conductor neutro ubicado en la parte inferior de las fases, la red puede soportar hasta $61 \mathrm{kA}$. Estos valores de intensidades de rayo significan las probabilidades de $33,58 \%$ y $14,68 \%$ que ocurran rayos iguales o mayores a $41 \mathrm{kA}$ y $61 \mathrm{kA}$ respectivamente. Esta evaluación se ha realizado considerando $5 \mathrm{Ohm}$ de resistencia de puesta a tierra en cada poste.

La anterior mejora explica que se debe ofrecer a la intensidad de corriente del rayo un camino de menor resistencia eléctrica para dispersarlo en el suelo antes de que la sobretensión que soportan los aisladores sea mayor al CFO y se origine descarga disruptiva en ellos; la mejor opción 
encontrada es la utilización del conductor de guarda junto al conductor neutro del sistema, permite en la práctica la conexión a tierra en paralelo de los dos conductores. En estas condiciones un impacto de rayo de $61 \mathrm{kA}$ descargado a tierra a través de una puesta a tierra de $5 \mathrm{Ohm}$ de resistencia eléctrica puede generar un potencial de hasta $296 \mathrm{kV}$ pico que presenta alto riesgo para las personas y animales respecto a las tensiones de toque y paso.

Teniendo en cuenta las anteriores observaciones, se ha analizado la utilización de aisladores ANSI clase 56-4 que tienen un CFO de $225 \mathrm{kV}$ positivo, los resultados demostraron mejor desempeño de la red ante descarga directa tipo rayo, principalmente cuando se considera el conductor de guarda en la red.

Se observa que las mayores magnitudes de corriente descargada en la puesta a tierra y el potencial generado en él, ocurren en la estructura más cercana al impacto del rayo, el poste contiguo ubicado a $180 \mathrm{~m}$ contribuye poco en la descarga de la corriente de rayo.

\section{CONCLUSIONES}

- Respecto a la primera hipótesis específica, el modelamiento del soporte, como impedancia de impulso y el aislamiento de la línea aérea de distribución rural con sus valores de CFO (Critical Impulse Flashover Voltage), logra respuestas correctas en la evaluación del desempeño ante descargas atmosféricas directa.

- Respecto a la primera hipótesis específica, el modelamiento del soporte, como impedancia de impulso y el aislamiento de la línea aérea de distribución rural con sus valores de CFO (Critical Impulse Flashover Voltage), logra respuestas correctas en la evaluación del desempeño ante descargas atmosféricas directas.

- Respecto de la segunda hipótesis específica, el modelo de la línea aérea de distribución rural aplicada al software EMTP RV, permite evaluar alternativas de mejora en el desempeño ante descargas atmosféricas como la inclusión de cable de guarda y valores diferentes de resistencia de puesta a tierra.

\section{RECOMENDACIONES}

- Es necesario implementar un bloque de simulación que enlace una descarga atmosférica a determinada distancia para poder evaluar el desempeño de la red ante sobretensiones inducidas.

- Se debe mejorar el modelamiento de los soportes de la red, considerando la contribución del CFO de las crucetas para evaluar si ello ayuda a mejorar el desempeño de la red ante descarga tipo rayo.

- La propuesta de cable de guarda, la utilización del conductor neutro del sistema y disminución de valores de resistencia de puesta a tierra debe ser evaluada económicamente, para su consideración en el costo de la red de distribución que directamente influye en la tarifa de energía eléctrica.

\section{REFERENCIAS BIBLIOGRÁFICAS}

Ministerio de Energía y Minas. R.D. N 016-2008-EM/DGE. Norma técnica de calidad de los servicios eléctricos rurales. Lima. Perú. 2008.

IEEE Std. 1410-2010, IEEE Guide for improving the lightning performance of electric power overhead distribution lines.

Electric Power Research Institute. Transmission line reference book, $345 \mathrm{kV}$ and Above. Palo Alto California. Second Edition. USA. 1982.

Caselles, A. Modelización y simulación de sistemas complejos. Publicación de la Universidad de Valencia. Edición digital. España. 2008.

Shannon, R. Introduction to the art and science of simulation. Proceedings of the 1998 winter simulation conference. USA- 1998 .

Sargent, M. \& Darveniza, M. “Tower surge impedance”, IEEE Transactions on power apparatus and systems, Vol. PAS88,pp. 680-687, May 1969

Ministerio de Energía y Minas. Norma DGE. "Especificaciones técnicas para el suministro de materiales y equipos de líneas y redes primarias para electrificación rural”. Lima. Perú. 2003.

Goni, M. y Ametani, A. "Analysis and estimation of surge impedance of tower”. ACES JOURNAL, Vol. 24, N. 1, February 2009.

Ministerio de Energía y Minas. Norma DGE. "Bases para el diseño de líneas y redes primarias para electrificación rural”. Lima. Perú. 2003.

ADINELSA. Línea de interconexión eléctrica en 22,9 kV Chirinos - Shumba Alto - Jaén. Expediente técnico. Cajamarca Perú. 2006. 


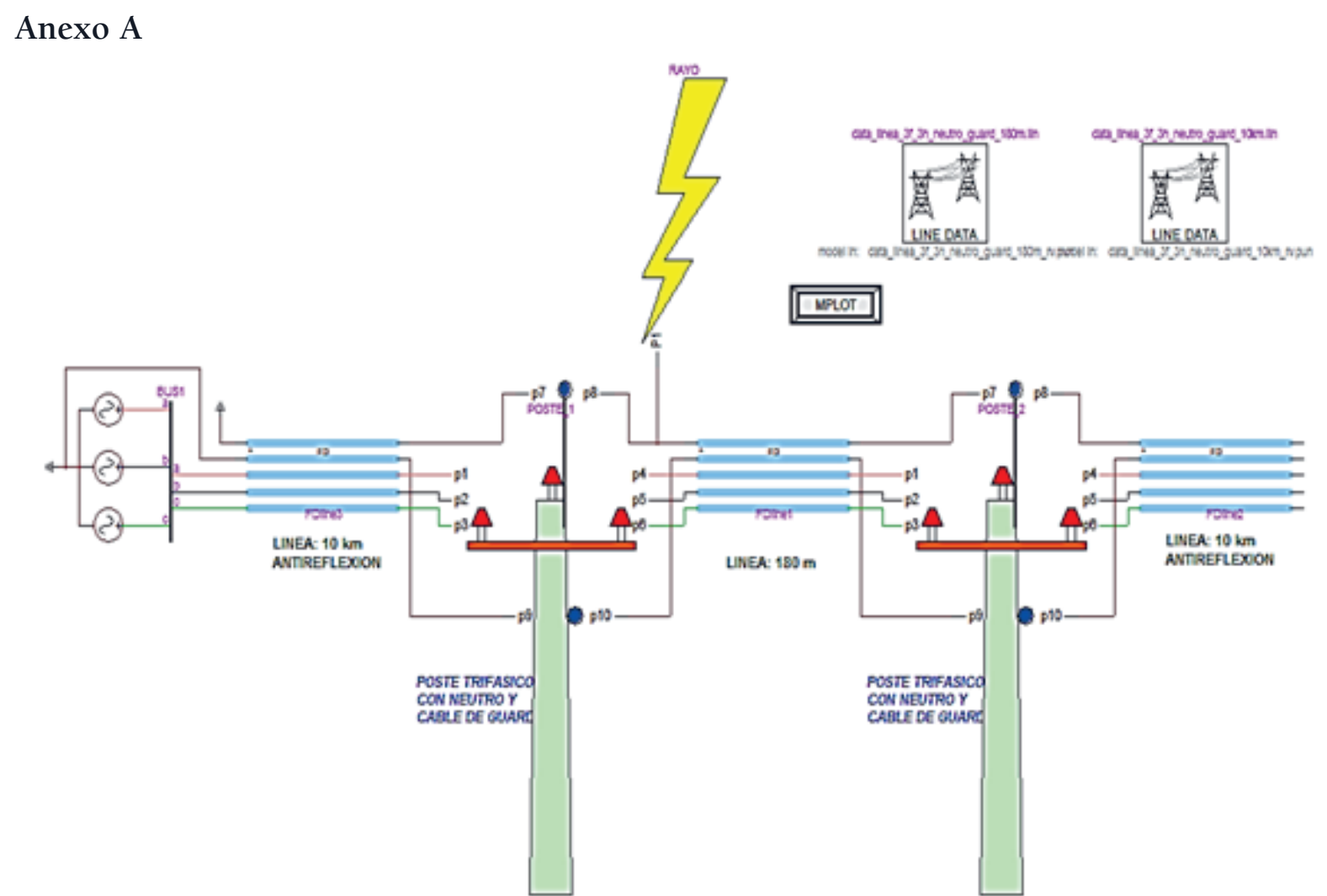

Figura 6. Modelización de red trifásica a cuatro hilos con conductor neutro y cable de guarda

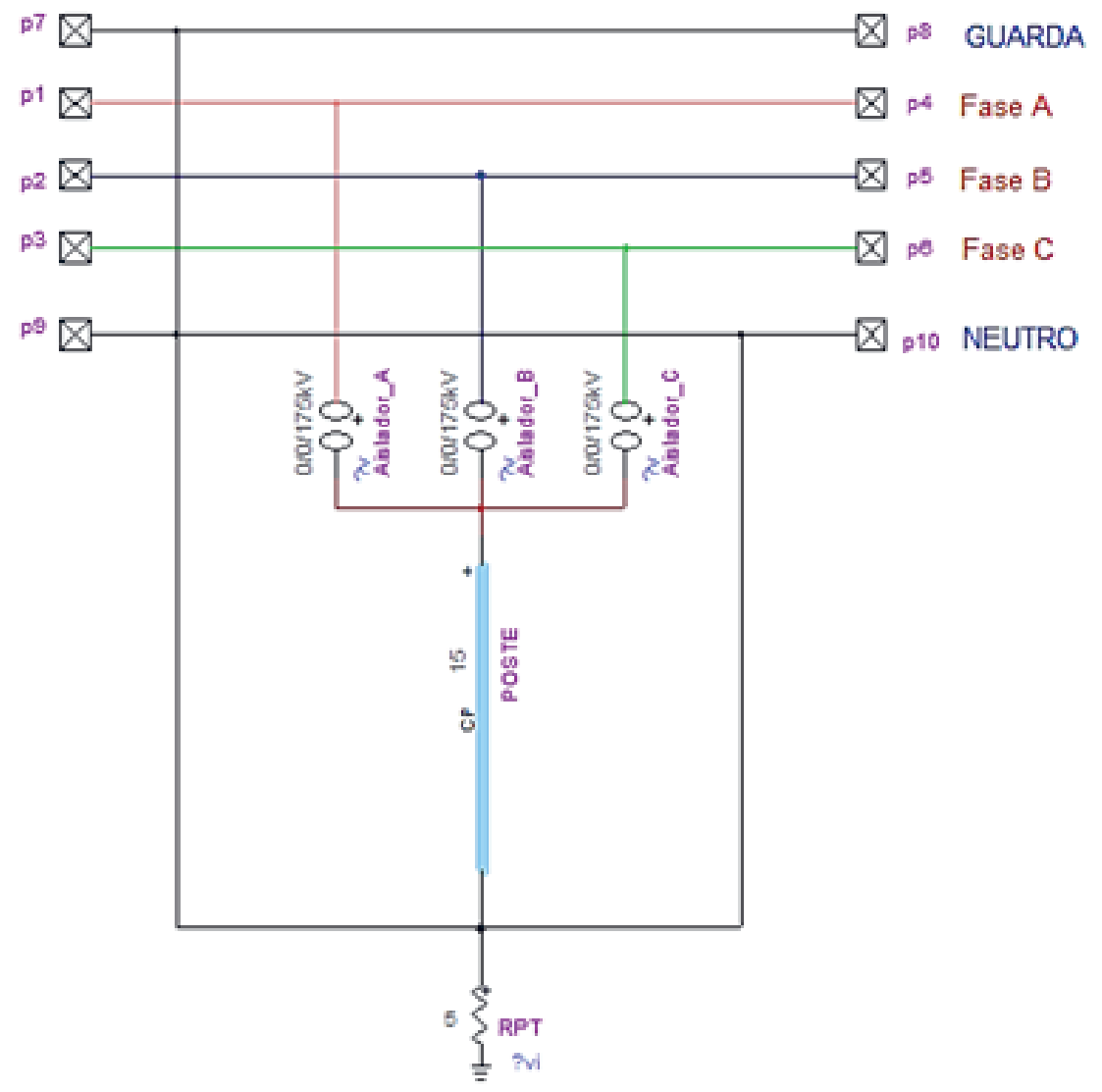

Figura 7. Modelización de la estructura de soporte 


\section{Anexo B}

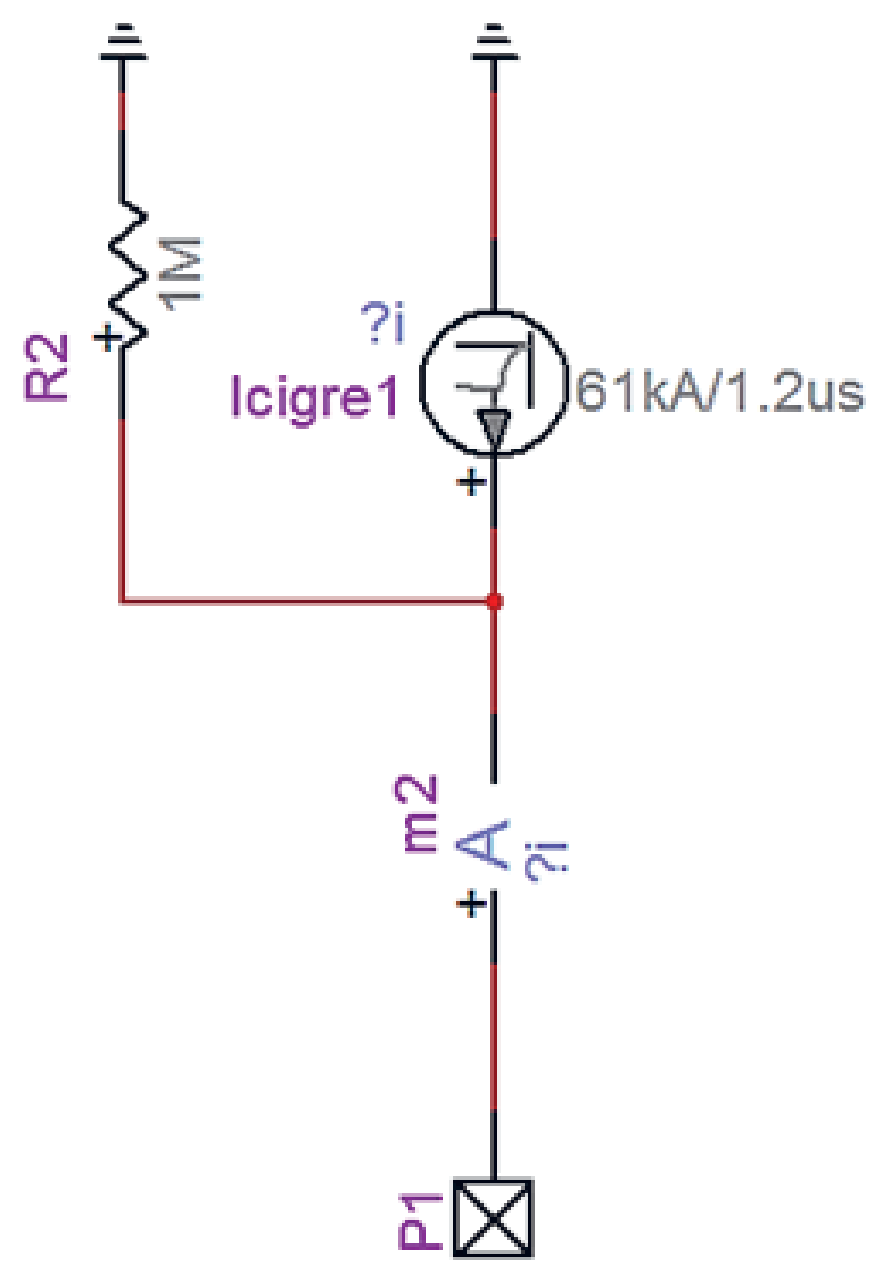

Figura 8. Modelización de la descarga atmosférica tipo rayo

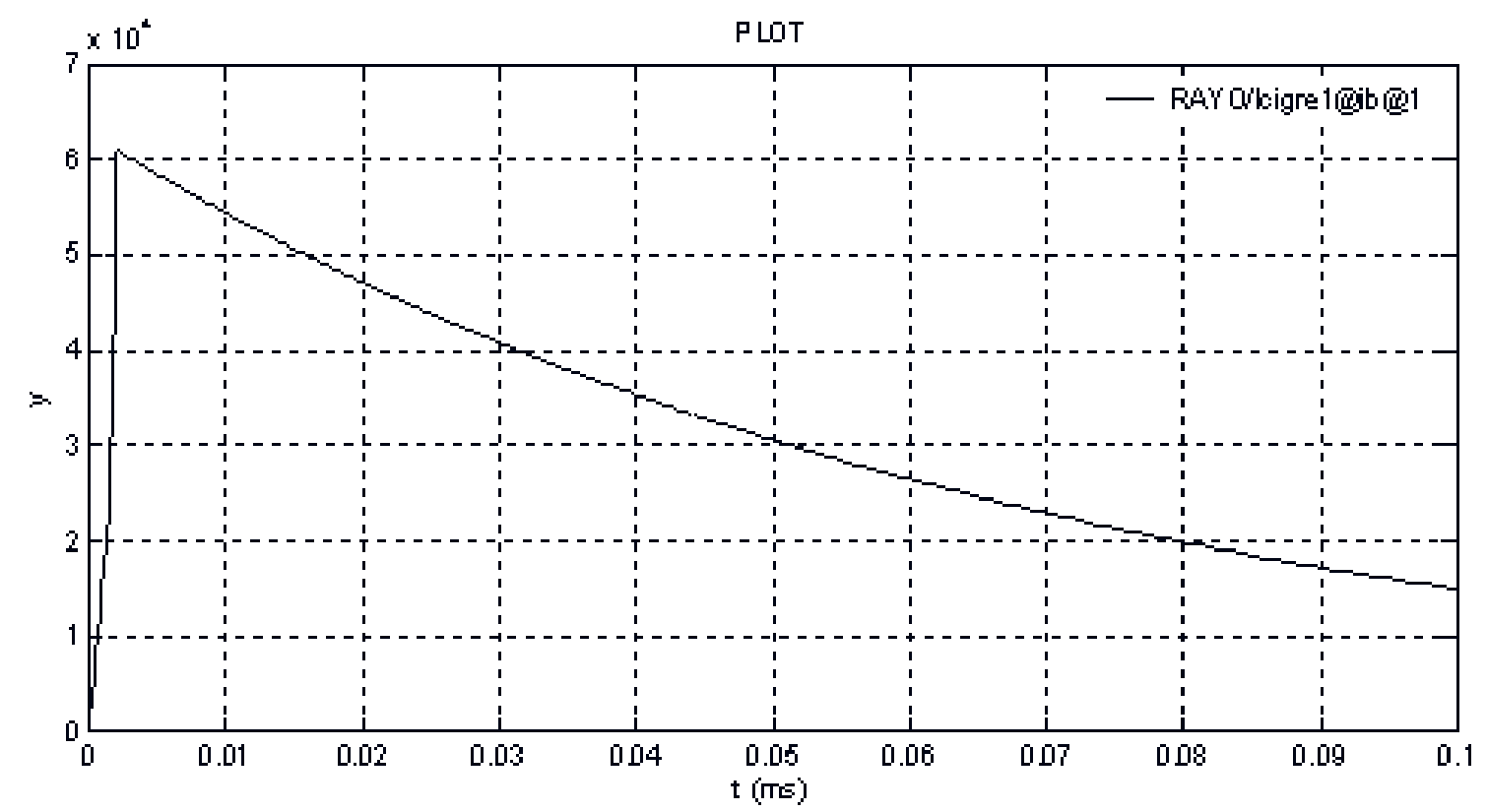

Figura 9. Onda de impulso de rayo normalizada: Respuesta del bloque de modelización de descarga atmosférica tipo rayo 


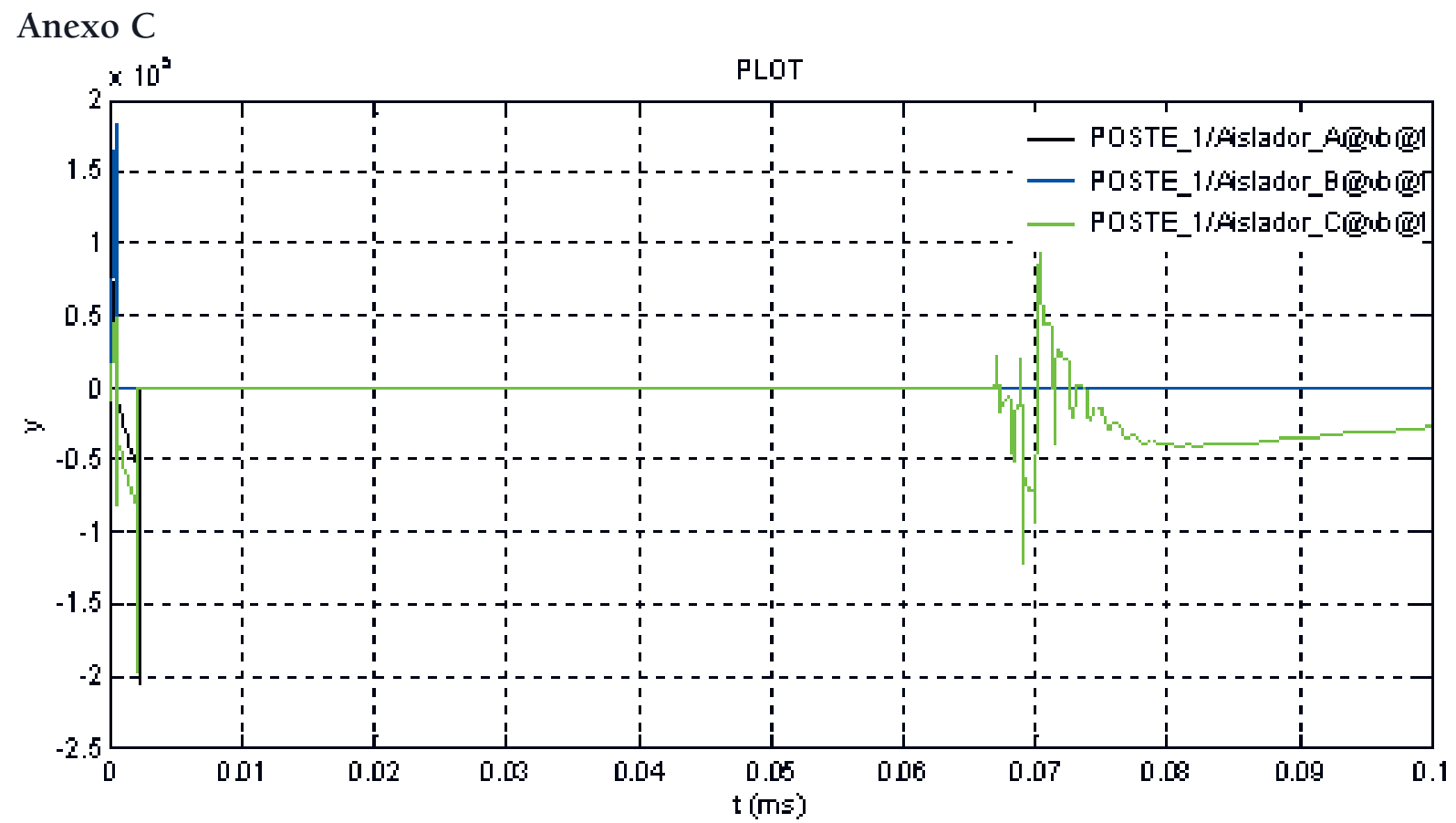

Figura 8. Respuesta de sobretensión en aisladores a causa de descarga directa de rayo de $10 \mathrm{kA}$ sobre la fase B en red trifásica a tres hilos. Ocurre flashover en las tres fases
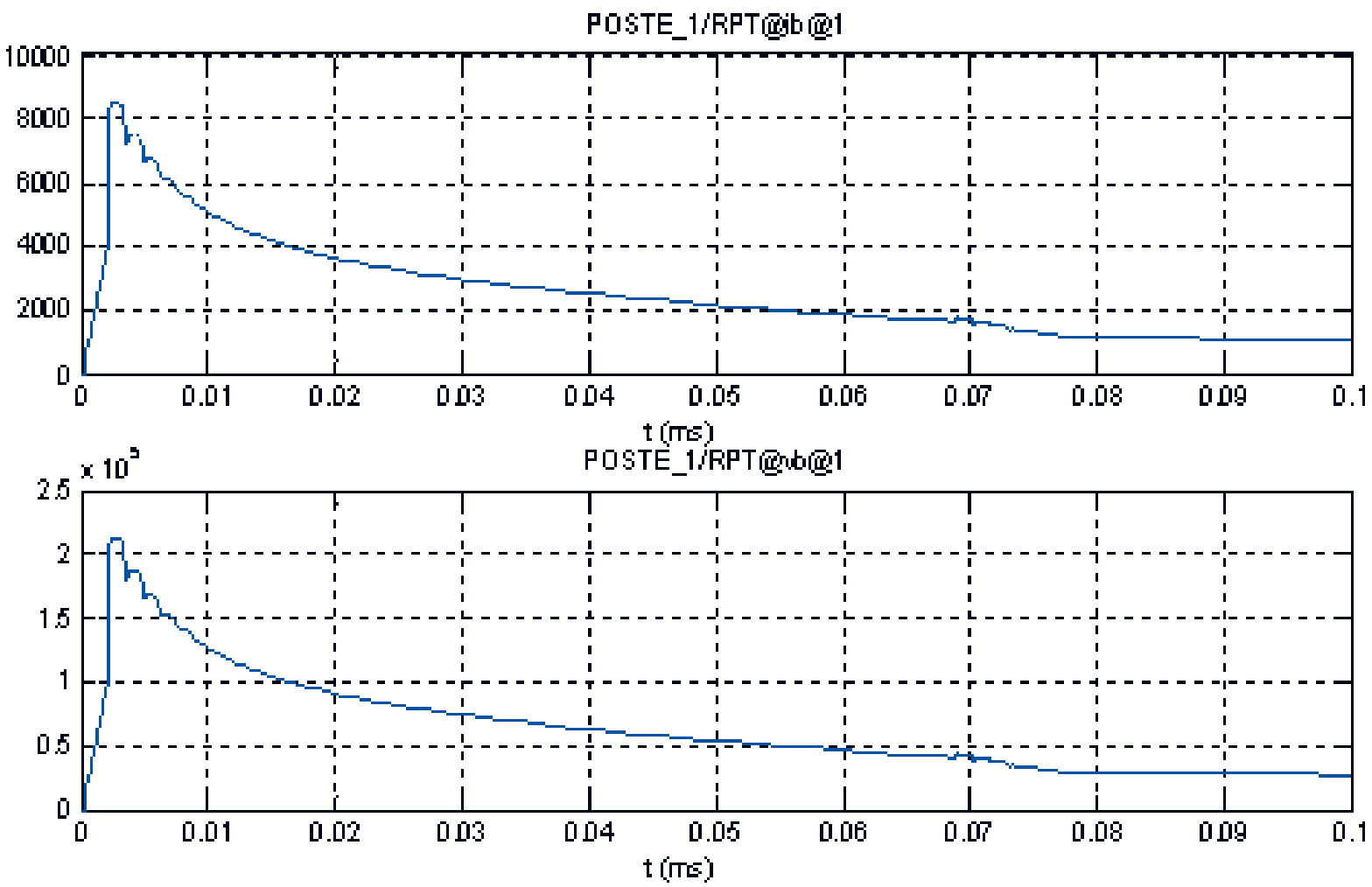

Figura 9. Formas de intensidad de corriente y potencial eléctrico en la puesta a tierra del poste 